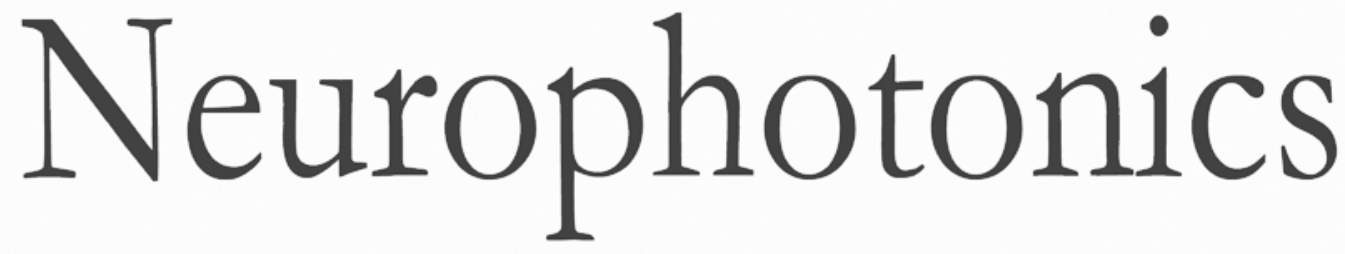

\title{
Publisher's note: False positives and false negatives in functional near- infrared spectroscopy: issues, challenges, and the way forward
}

Ilias Tachtsidis

Felix Scholkmann 


\title{
Publisher's note: False positives and false negatives in functional near-infrared spectroscopy: issues, challenges, and the way forward
}

\author{
Ilias Tachtsidis ${ }^{a}$ and Felix Scholkmann ${ }^{b, *}$ \\ aUniversity College London, Department of Medical Physics and Biomedical Engineering, Gower Street, \\ Malet Place Engineering Building, WC1E 6BT, London, United Kingdom \\ bUniversity Hospital Zurich, University of Zurich, Department of Neonatology, Biomedical Optics Research Laboratory, \\ Frauenklinikstr. 10, 8091 Zurich, Switzerland
}

[DOI: 10.1117/1.NPh.3.3.039801]

This article [Neurophoton. 3(3), 030401 (2016)] was originally published with an erroneous citation identifier (CID). It was republished online with a corrected CID of 031405 on 15 March 2016. The article appears correctly in print. 